\title{
Factors associated with quitting status of smoking in Korean men with and without chronic kidney disease: A national population-based study
}

\author{
Youngmee Kim ${ }^{1}$, Won-Kyung Cho ${ }^{2,3}$
}

\begin{abstract}
INTRODUCTION Adverse effects of smoking on kidney function have been demonstrated in both general populations and in populations with chronic kidney disease (CKD). Therefore, quitting smoking can have a significant impact on the mortality and disease progression of CKD. This study examined and compared factors associated with quitting status of smoking, in patients with and without CKD, among Korean adult male smokers, using the Korea National Health and Nutrition Examination Survey from 2008 to 2019 , excluding 2013.

METHODS Wald test with multiple logistic regression was performed to investigate factors associated with quitting smoking in both CKD and non-CKD groups, along with the interaction effects between groups.

RESULTS Of the 15747 eligible individuals, 909 had CKD, of whom 703 (weighted percentage: $74.4 \%$ ) were quitters. In the non-CKD group, 8393 (weighted percentage: $50.4 \%$ ) succeeded in quitting. Regular exercise was the only factor associated with quitting in both groups. The adjusted odd ratios with confidence intervals were 1.29 (95\% CI: $1.17-1.42)$ and 2.84 (95\% CI: $1.52-5.31$ ) in the non-CKD and CKD groups, respectively (interaction $\mathrm{p}=0.0153$ ). Unlike in the CKD group, marriage and higher systolic blood pressure were also associated with quitting, and lifetime smoking amount and secondhand smoke exposure at home were negatively associated with smoking cessation in the non-CKD group. CONCLUSIONS Exercise was the only factor associated with quitting smoking in the CKD group.
\end{abstract}

\author{
AFFILIATION \\ 1 Red Cross College of \\ Nursing, Chung-Ang \\ University, Seoul, Republic \\ of Korea \\ 2 Department of \\ Pulmonology and Critical \\ Care Medicine, Asan Medical \\ Center, University of Ulsan, \\ Seoul, Republic of Korea \\ 3 International Healthcare \\ Center, Asan Medical Center, \\ University of Ulsan, Seoul, \\ Republic of Korea

\section{CORRESPONDENCE TO} \\ Won-Kyung Cho. Asan \\ Medical Center, University of \\ Ulsan College of Medicine, \\ Olympic-ro 43-gil, Songpa- \\ gu, 05505, Seoul, Republic \\ of Korea. \\ E-mail:wonkyungcho@ \\ hotmail.com \\ ORCID ID: https://orcid. \\ org/0000-0003-1540-545X \\ KEYWORDS \\ chronic kidney disease, CKD, \\ Koreans, male smokers, \\ smoking cessation
}

Received: 21 September 2021

Revised: 30 November 2021

Accepted: 11 January 2022

\section{INTRODUCTION}

Chronic kidney disease (CKD) is defined as kidney damage or decreased kidney function for more than three months ${ }^{1}$. It is estimated that about $9 \%$ of the population is affected by CKD worldwide. In 2017, CKD resulted in 1.2 million deaths and was the 12th leading cause of death worldwide. The significant causes of death in the CKD population are disease progression or CKD-attributable cardiovascular disease $(\mathrm{CVD})^{2}$. High cardiovascular mortality in this population can be partly explained by the frequent occurrence of CKD in diseases associated with cardiovascular risk, such as hypertension and diabetes. However, CKD is also an independent risk factor for cardiovascular death ${ }^{1,3,4}$. Smoking tobacco is a leading cause of CVD morbidity and mortality ${ }^{5}$. 
This means that patients with CKD, who already have a higher CVD risk than the general population, are at an even greater risk if they smoke.

CKD may progress to end-stage kidney disease. Some patients with CKD demonstrate a more rapid progression rate, while others have relatively stable disease. Various factors have been suggested as contributors to disease progression in patients with CKD or modifiers of the disease course, such as comorbid conditions, treatments, socioeconomic status, and ethnicity ${ }^{6-8}$. Adverse effects of smoking on kidney function have been demonstrated in both the general population and CKD-specific populations. Specifically, it has been reported that albuminuria, nephrosclerosis or glomerulonephritis are more common in smokers ${ }^{8-15}$. The mechanisms of smoking-related kidney damage can be explained by hemodynamic or non-hemodynamic effects, such as nicotine toxicity, oxidative stress, thrombotic, and inflammatory cytokine release ${ }^{9}$. This means that smoking can modify the disease course of CKD. Indeed, a few studies have suggested that smoking cessation may slow CKD progression ${ }^{9,16,17}$. These findings indicate that quitting smoking can significantly impact the mortality and disease progression of CKD. Many smokers, aware of tobacco's harmfulness, want to quit but often fail. Considering how difficult it is to quit smoking, identifying the factors involved is the first step in helping people to do so.

Numerous physiological, behavioral, environmental, psychological, cognitive, and social factors are involved in the success or failure of smoking cessation in the general population ${ }^{18-21}$. However, there have been no studies to examine CKD-specific factors related to smoking cessation. In this context, this study examined and compared the quitting status of smoking and the factors associated with quitting status in study participants with and without CKD among Korean adult male smokers using nationally representative data, the Korea National Health and Nutrition Examination Survey (KNHANES).

\section{METHODS}

\section{Study design and ethical considerations}

This study was conducted using data from the Korea National Health and Nutrition Examination Survey (KNHANES) from 2008 to 2019, excluding 2013 because it did not contain detailed information on smoking cessation. The KNHANES is an ongoing, annual, nationwide, population-based, cross-sectional, multistage, stratified, and clustered probability sampling survey based on geographical area, age, and gender by the Korea Centers for Disease Control and Prevention (KCDC), which examines the health, lifestyle, and eating habits of Koreans ${ }^{22}$. The data consist of questionnaires on physical and mental health and laboratory tests. KNHANES consists of three components: a health interview, health examination, and nutrition survey. The health interview and health examination are conducted by trained medical staff and interviewers at the mobile examination center. A week after the health interview and examination surveys, dieticians visit the participants' homes for the nutrition survey. Also, blood samples are collected at the mobile examination centers in the morning after a fast by participants of at least 8 hours for the collection of laboratory data.

The data were downloaded after the registration process designated for accessing the official KNHANES website (http://knhanes.cdc.go.kr/). Before the survey, written informed consent was obtained from each study participant ${ }^{22}$. The current study used only existing de-identified data. The study was conducted according to the guidelines of the Declaration of Helsinki. The institutional review board (IRB) of the Korea Centers for Disease Control and Prevention (KCDC) reviewed and approved the KNHANES survey. The IRB approval numbers were 2008-04EXP-01-C, 2009-01 CON03-2C, 2010-02CON-21-C, 2011-02CON-06-C, 2012-01EXP-01-2C, 2013-12EXP-03-5C, 2018-0103-P-A, and 2018-01-03-C-A.

\section{Participants}

The inclusion criteria of the present study were men aged $\geq 19$ years having serum creatinine results, who have smoked more than 100 cigarettes in their lifetime, and have tried to quit smoking in the past. We excluded: 1) never smokers or persons with a lifetime smoking history of $<100$ cigarettes; and 2) those who did not attempt to quit smoking. A total of 93120 people were screened, and finally, 15747 people were included in the study, according to the inclusion criteria. The final sample comprised 14838 men without CKD and 909 men with CKD.

This study included only men because smoking 
history was examined using only self-report data. Previous studies showed a significant difference between self-reported smoking rates and those assessed by measurement of cotinine level in Korean women, which is thought to be related to social stigma and prejudice they face ${ }^{23-25}$. Due to the limited availability of cotinine levels in the KNHANES data, we have decided to include only men in the analysis.

Figure 1 demonstrates the flow diagram of the selection process and the number of study participants.

\section{Defining and measuring important variables}

In this study, CKD was defined as an estimated glomerular filtration rate $(\mathrm{eGFR})<60 \mathrm{~mL} / \mathrm{min} / 1.73$ $\mathrm{m}^{2}$. The eGFR was calculated using the Modification of Diet in Renal Disease equation ${ }^{26,27}$.

Quitters were defined as those who reported having smoked more than 100 cigarettes in their lifetime and who were no longer smokers at the time of the survey. Non-quitters were those who reported having smoked more than 100 cigarettes in their lifetime and were smokers at the time of the study, with unsuccessful past attempts to quit smoking ${ }^{28,29}$. Our definitions of smoker, non-smoker, quitter, and non-quitter, are based on the CDC recommendations ${ }^{28,29}$.

Other than current smoking status, additional smoking-related histories such as lifetime smoking amount (pack-years), exposure to secondhand smoke, and smoking cessation methods were obtained using a questionnaire. Regular exercise was defined as exercising at a vigorous intensity for 20 or more minutes per session at least three times a week or exercising at moderate intensity for 30 or more minutes per session at least five times a week, performing vigorous exercise for 1.25 hours or more a week, or a moderate level of exercise for 2.5 hours

Figure 1. Flow diagram showing participant selection and the numbers of participants

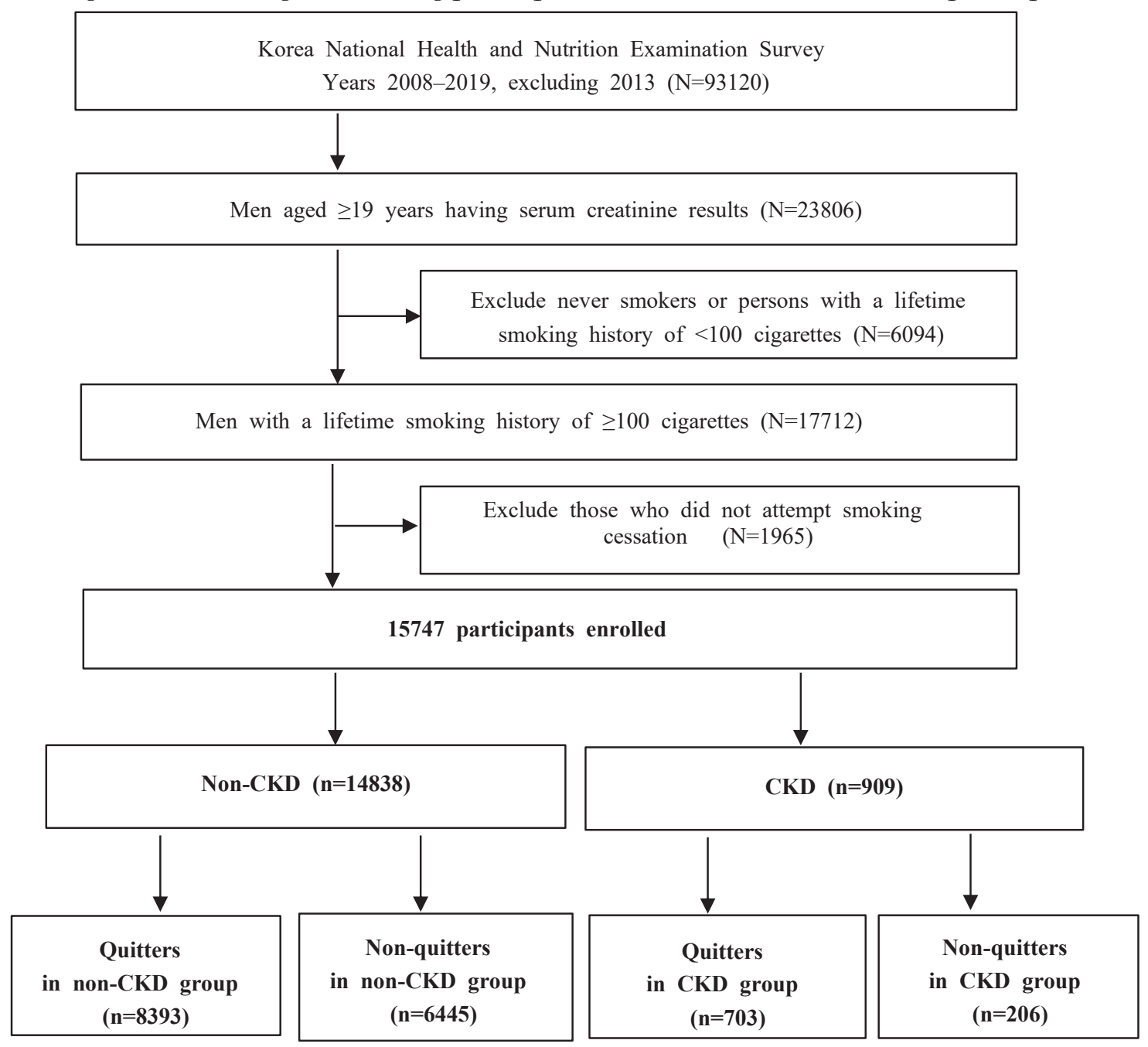


or more a week. Participants were advised to answer exercise intensity according to the subjective degree of body's hardship and shortness of breath. To help participants better understand the categories, some examples were also provided in the questionnaire. For example, running, swimming, and lifting heavy objects are regarded as vigorous exercises. Table tennis and lifting light objects are considered moderate exercises.

Trauma history was defined as a history of at least one accident or intoxication that required hospitalization and/or emergency room treatment during the past year. Heavy drinkers were defined as those who consume seven or more glasses of alcohol (regardless of alcohol type) per occasion at least twice per week. Perceived health status was defined as an individual's perceived overall health level (i.e. very good/good, fair, or poor/very poor). Perceived psychological stress was defined as moderate-tosevere daily stress ${ }^{22,30}$.

\section{Statistical analysis}

All data analyses were conducted using SAS version 9.4 (SAS Institute, Inc., Cary, NC, USA) and presented as mean \pm standard error (SE) for continuous variables or as proportions $( \pm \mathrm{SE})$ for categorical variables. The prevalence of quitters and non-quitters according to the absence and presence of CKD was presented as a proportion $( \pm \mathrm{SE})$ (Table 1$)$. T-tests and chi-squared tests were conducted to evaluate the differences between groups for continuous and categorical variables, respectively (Tables 2 and 3 ).

Variables with $\mathrm{p}<0.1$ in Tables 2 and 3 were used for the multiple logistic regression. Wald test with multiple logistic regression analyses was performed to investigate factors associated with smoking cessation in both CKD and non-CKD groups and the interaction effects between the groups. All estimates for all interaction terms between CKD and non-CKD groups were obtained from the multiple logistic regression adjusting for the involved characteristics such as age, marital status, household income, lifetime smoking amount, secondhand smoke exposure, and smoking cessation methods. Diastolic blood pressure and waist circumference were omitted from the analysis because of multi-collinearity. Drinking coffee was also omitted because it was surveyed only from 2012-2016. A p $<0.05$ or $95 \%$ confidence interval (CI) that did not span 1.0 was considered statistically significant (Table 4). As KNHANES' data are derived from stratified and multistage clustered probability sampling methods to represent the entire Korean population, population weights were applied to the analyses ${ }^{31}$.

Table 1. Prevalence of quitters and non-quitters according to non-CKD and/or CKD groups (N=15747)

\begin{tabular}{|c|c|c|c|c|c|c|c|c|c|}
\hline \multirow[t]{2}{*}{ Year } & \multicolumn{3}{|c|}{ Total $(\mathbf{N}=15747)$} & \multicolumn{3}{|c|}{ Non-CKD $(n=14838)$} & \multicolumn{3}{|c|}{ CKD $(n=909)$} \\
\hline & $\begin{array}{c}\text { Quitter } \\
(n=9096)^{a} \\
\%(S E)\end{array}$ & $\begin{array}{l}\text { Non-quitter } \\
\begin{array}{c}(n=665 I)^{b} \\
\%(\mathrm{SE})\end{array}\end{array}$ & $p$ & $\begin{array}{c}\text { Quitter } \\
(n=8393)^{c} \\
\%(\mathrm{SE})\end{array}$ & $\begin{array}{l}\text { Non-quitter } \\
\begin{array}{c}(n=6445)^{d} \\
\%(\mathrm{SE})\end{array}\end{array}$ & $p$ & $\begin{array}{l}\text { Quitter } \\
(n=703)^{\mathrm{e}} \\
\%(\mathrm{SE})\end{array}$ & $\begin{array}{l}\text { Non-quitter } \\
\qquad \begin{array}{c}(n=206)^{r} \\
\%(\mathrm{SE})\end{array}\end{array}$ & $p$ \\
\hline 2008 & $48.7(1.47)$ & $51.3(1.47)$ & $<0.0001$ & $47.3(1.50)$ & $52.7(1.50)$ & $<0.0001$ & $75.4(5.31)$ & $24.6(5.31)$ & 0.0309 \\
\hline 2009 & $46.1(1.35)$ & $53.9(1.35)$ & & $45.3(1.37)$ & $54.7(1.37)$ & & $75.7(6.03)$ & $24.3(6.03)$ & \\
\hline 2010 & $46.3(1.76)$ & $53.7(1.76)$ & & $45.3(1.81)$ & $54.7(1.81)$ & & 78.0 (5.88) & $22.0(5.88)$ & \\
\hline 2011 & 47.9 (1.68) & $52.1(1.68)$ & & $46.4(1.71)$ & $53.6(1.71)$ & & $81.0(5.90)$ & $19.0(5.90)$ & \\
\hline 2012 & $50.6(1.61)$ & $49.4(1.61)$ & & $50.3(1.64)$ & $49.7(1.64)$ & & $60.4(7.75)$ & 39.6 (7.75) & \\
\hline 2014 & $47.6(2.04)$ & $52.4(2.04)$ & & $46.9(2.07)$ & $53.1(2.07)$ & & $70.6(7.49)$ & $29.4(7.49)$ & \\
\hline 2015 & $54.7(1.51)$ & $45.3(1.51)$ & & $53.7(1.58)$ & $46.3(1.58)$ & & $78.1(5.46)$ & $21.9(5.46)$ & \\
\hline 2016 & $55.0(1.79)$ & $45.0(1.79)$ & & $53.5(1.85)$ & $46.5(1.85)$ & & $88.7(3.38)$ & $11.3(3.38)$ & \\
\hline 2017 & $57.4(1.68)$ & $42.6(1.68)$ & & $56.9(1.73)$ & $43.1(1.73)$ & & $69.5(5.90)$ & $30.5(5.90)$ & \\
\hline 2018 & $59.3(1.50)$ & $40.7(1.50)$ & & $58.6(1.56)$ & $41.4(1.56)$ & & $79.4(5.35)$ & $20.6(5.35)$ & \\
\hline 2019 & $59.1(1.48)$ & 40.9 (1.48) & & $58.8(1.52)$ & $41.2(1.52)$ & & $63.4(5.93)$ & $36.6(5.93)$ & \\
\hline
\end{tabular}

Data have been presented as weighted percentages (standard error, SE). The p-value was determined using the Rao-Scott chi-squared test. Data were not collected in 2013. a Weighted $n$ (weighted \%)=6214858 (51.3\%). b Weighted $n=5895954(48.7 \%)$. c Weighted $n=5876685(50.4 \%)$. d Weighted $n=5779842(49.6 \%)$. e Weighted $n=338173(74.4 \%)$. f Weighted $n=116112$ (25.6\%). CKD: chronic kidney disease. 


\section{RESULTS}

\section{Prevalence of quitters and non-quitters}

Table 1 displays the prevalence rates of quitters and nonquitters of the non-CKD and the CKD groups from 2008 to 2019, excluding 2013. Among the 15747 participants, 909 individuals suffered from CKD. Of the 14838 individuals in the non-CKD group, 8393 (weighted percentage: $50.4 \%$ ) succeeded in quitting. Among the
909 individuals in the CKD group, 703 (weighted percentage: $74.4 \%$ ) were quitters. In the non-CKD group, the success rates of smoking cessation tended to increase during the study period. The success rates showed slight fluctuations over the study period in the CKD group but were generally steady. The success rates of the CKD group were consistently higher than those in the non-CKD group throughout the study period.

Table 2. Sociodemographic characteristics and smoking history of participants (N=15747)

\begin{tabular}{|c|c|c|c|c|c|c|}
\hline \multirow[t]{2}{*}{ Variables } & \multicolumn{3}{|c|}{ Non-CKD $(n=14838)$} & \multicolumn{3}{|c|}{ CKD $(n=909)$} \\
\hline & $\begin{array}{l}\text { Quitter } \\
(n=8393) \\
\%(S E)\end{array}$ & $\begin{array}{c}\text { Non-quitter } \\
(n=6445) \\
\%(\mathrm{SE})\end{array}$ & $p$ & $\begin{array}{l}\text { Quitter } \\
(n=703) \\
\%(\text { SE })\end{array}$ & $\begin{array}{l}\text { Non-quitter } \\
\qquad \begin{array}{c}(n=206) \\
\%(S E)\end{array}\end{array}$ & $p$ \\
\hline Age (years), mean $\pm \mathrm{SE}$ & $51.65 \pm 0.20$ & $42.08 \pm 0.21$ & $<0.0001$ & $67.72 \pm 0.58$ & $61.78 \pm 1.16$ & $<0.0001$ \\
\hline Age (years) & & & $<0.0001$ & & & $<0.0001$ \\
\hline $19-39$ & $21.6(0.63)$ & $46.3(0.81)$ & & $1.4(0.75)$ & $3.8(2.27)$ & \\
\hline $40-64$ & $57.2(0.67)$ & $47.5(0.78)$ & & $32.0(2.33)$ & $53.7(4.38)$ & \\
\hline$\geq 65$ & $21.2(0.51)$ & $6.2(0.28)$ & & $66.7(2.35)$ & $42.5(4.18)$ & \\
\hline Married & $84.3(0.52)$ & $65.4(0.80)$ & $<0.0001$ & $84.8(1.82)$ & $81.4(3.62)$ & 0.3709 \\
\hline Education level & & & 0.4447 & & & 0.1446 \\
\hline$\leq$ High school & $60.1(0.78)$ & $60.8(0.77)$ & & $80.2(1.94)$ & $73.7(4.30)$ & \\
\hline University or higher & $39.9(0.78)$ & $39.2(0.77)$ & & $19.8(1.94)$ & $26.3(4.30)$ & \\
\hline Occupation & & & $<0.0001$ & & & 0.0009 \\
\hline Manager/professionals & $16.7(0.57)$ & $16.8(0.57)$ & & $5.9(1.32)$ & $17.1(3.96)$ & \\
\hline Office worker & $12.7(0.46)$ & $12.9(0.50)$ & & $4.9(1.13)$ & $2.5(1.10)$ & \\
\hline Service workers/sellers & $10.9(0.44)$ & $14.8(0.56)$ & & $5.6(1.21)$ & $8.9(2.86)$ & \\
\hline Agriculture/fishery/labor & $36.4(0.71)$ & $37.2(0.75)$ & & $24.1(2.04)$ & $23.8(3.64)$ & \\
\hline None & $23.2(0.58)$ & $18.3(0.61)$ & & $59.5(2.40)$ & $47.7(4.60)$ & \\
\hline Residence (\% rural) & $17.3(0.88)$ & $17.8(0.98)$ & 0.5173 & $22.0(2.05)$ & $23.5(3.76)$ & 0.7086 \\
\hline Household income (quartiles) & & & $<0.0001$ & & & $<0.0001$ \\
\hline 1st (lowest) & $21.8(0.57)$ & $26.7(0.70)$ & & $22.2(1.91)$ & $41.4(4.27)$ & \\
\hline 2nd & $25.3(0.59)$ & $26.1(0.67)$ & & $25.3(2.09)$ & $23.3(3.67)$ & \\
\hline $3 r d$ & $25.5(0.57)$ & $25.0(0.67)$ & & $23.8(2.03)$ & $17.4(3.12)$ & \\
\hline 4th (highest) & $27.5(0.66)$ & $22.2(0.69)$ & & $28.7(2.10)$ & $17.9(3.24)$ & \\
\hline $\begin{array}{l}\text { Lifetime smoking amount (pack-years), } \\
\text { mean } \pm \text { SE }\end{array}$ & $18.08 \pm 0.22$ & $17.51 \pm 0.22$ & 0.0529 & $28.21 \pm 1.09$ & $25.02 \pm 1.17$ & 0.0439 \\
\hline \multicolumn{7}{|l|}{ Secondhand smoke exposure } \\
\hline Workplace (yes) & $30.1(0.66)$ & $41.3(0.76)$ & $<0.0001$ & $14.0(1.74)$ & $23.0(4.18)$ & 0.0269 \\
\hline Home (yes) & $2.4(0.21)$ & $7.6(0.44)$ & $<0.0001$ & $4.0(1.11)$ & $2.8(1.09)$ & 0.4577 \\
\hline \multicolumn{7}{|l|}{ Smoking cessation methods } \\
\hline Willpower & $93.6(0.32)$ & $84.4(0.52)$ & $<0.0001$ & $94.9(1.06)$ & $88.3(2.65)$ & 0.0066 \\
\hline Nicotine replacement therapy & $6.0(0.32)$ & $17.3(0.55)$ & $<0.0001$ & $4.9(1.08)$ & $10.0(2.53)$ & 0.0347 \\
\hline Education/counselling & $5.2(0.29)$ & $11.9(0.45)$ & $<0.0001$ & $4.0(0.84)$ & $12.9(2.69)$ & $<0.0001$ \\
\hline Smokers' quitline & $0.6(0.10)$ & $1.4(0.16)$ & $<0.0001$ & $0.3(0.22)$ & $1.4(0.85)$ & 0.0669 \\
\hline
\end{tabular}

Data are presented as weighted mean \pm standard error (SE) or weighted percent (SE). The p-values were determined by Student's t-test or Rao-Scott chi-squared test. Income quartiles are age and gender adjusted. CKD: chronic kidney disease. 
Table 3. Clinical characteristics, health behaviors, and perceived health ( $N=15747)$

\begin{tabular}{|c|c|c|c|c|c|c|}
\hline \multirow[t]{2}{*}{ Variables } & \multicolumn{3}{|c|}{ Non-CKD ( $n=14838)$} & \multicolumn{3}{|c|}{ CKD $(n=909)$} \\
\hline & $\begin{array}{c}\text { Quitter } \\
(n=8393) \\
\%(\mathrm{SE})\end{array}$ & $\begin{array}{c}\text { Non-quitter } \\
\begin{array}{c}(n=6445) \\
\%(S E)\end{array}\end{array}$ & $p$ & $\begin{array}{l}\text { Quitter } \\
(n=703) \\
\%(S E)\end{array}$ & $\begin{array}{c}\text { Non-quitter } \\
\begin{array}{c}(n=206) \\
\%(S E)\end{array}\end{array}$ & $p$ \\
\hline \multicolumn{7}{|l|}{ Health status } \\
\hline eGFR, mean $\pm \mathrm{SE}$ & $86.16 \pm 0.20$ & $90.94 \pm 0.23$ & $<0.0001$ & $50.41 \pm 0.53$ & $50.12 \pm 0.98$ & 0.7943 \\
\hline eGFR $\geq 90$ & $35.9(0.68)$ & $49.7(0.78)$ & $<0.0001$ & - & - & 0.4441 \\
\hline eGFR 89-60 & $64.1(0.68)$ & $50.3(0.78)$ & & - & - & \\
\hline CKD Stage $3^{\text {a }}$ & - & - & & $79.6(1.91)$ & $77.2(3.50)$ & \\
\hline CKD Stage $3^{b}$ & - & - & & $15.3(1.66)$ & $14.7(2.90)$ & \\
\hline CKD Stage $4-5$ & - & - & & $5.2(1.14)$ & $8.1(2.36)$ & \\
\hline Systolic BP $(\mathrm{mmHg})$, mean $\pm \mathrm{SE}$ & $121.36 \pm 0.21$ & $117.86 \pm 0.22$ & $<0.0001$ & $127.16 \pm 0.80$ & $126.95 \pm 1.59$ & 0.9075 \\
\hline Diastolic BP $(\mathrm{mmHg})$, mean $\pm \mathrm{SE}$ & $78.86 \pm 0.15$ & $78.03 \pm 0.17$ & $<0.0001$ & $74.97 \pm 0.55$ & $76.49 \pm 1.21$ & 0.2524 \\
\hline Body mass index $\left(\mathrm{kg} / \mathrm{m}^{2}\right)$, mean $\pm \mathrm{SE}$ & $24.52 \pm 0.04$ & $24.27 \pm 0.05$ & $<0.0001$ & $24.52 \pm 0.13$ & $24.47 \pm 0.32$ & 0.8899 \\
\hline Waist circumference $(\mathrm{cm})$, mean $\pm \mathrm{SE}$ & $86.42 \pm 0.12$ & $85.07 \pm 0.14$ & $<0.0001$ & $88.30 \pm 0.41$ & $88.00 \pm 0.83$ & 0.7511 \\
\hline Fasting blood sugar $(\mathrm{mg} / \mathrm{dL})$, mean $\pm \mathrm{SE}$ & $103.00 \pm 0.29$ & $99.62 \pm 0.35$ & $<0.0001$ & $109.67 \pm 1.33$ & $112.37 \pm 3.43$ & 0.4654 \\
\hline Total cholesterol $(\mathrm{mg} / \mathrm{dL})$, mean $\pm \mathrm{SE}$ & $190.46 \pm 0.49$ & $190.46 \pm 0.55$ & 0.9901 & $177.97 \pm 1.75$ & $182.41 \pm 4.26$ & 0.3449 \\
\hline Hypertension & $35.9(0.62)$ & $23.3(0.63)$ & $<0.0001$ & $74.5(2.04)$ & $73.3(3.81)$ & 0.7895 \\
\hline Diabetes mellitus & $13.0(0.42)$ & $9.3(0.40)$ & $<0.0001$ & $31.5(2.14)$ & $38.5(4.41)$ & 0.1417 \\
\hline Cardiovascular disease & $5.6(0.26)$ & $2.6(0.20)$ & $<0.0001$ & $23.8(1.96)$ & $12.2(2.53)$ & 0.0012 \\
\hline Cancer & $4.3(0.23)$ & $1.2(0.14)$ & $<0.0001$ & 9.1 (1.28) & $3.8(1.46)$ & 0.0243 \\
\hline Trauma history & $7.2(0.36)$ & $9.6(0.45)$ & $<0.0001$ & $5.4(1.12)$ & $7.2(2.01)$ & 0.4291 \\
\hline Heavy drinking & $19.7(0.54)$ & $29.3(0.67)$ & $<0.0001$ & $6.4(1.17)$ & $9.2(2.34)$ & 0.2420 \\
\hline Regular exercise & $41.8(0.70)$ & $37.7(0.74)$ & $<0.0001$ & $30.5(2.16)$ & 20.7 (3.74) & 0.0403 \\
\hline Sleep duration (h/day), mean \pm SE & $6.96 \pm 0.02$ & $6.98 \pm 0.02$ & 0.4065 & $7.06 \pm 0.09$ & $7.09 \pm 0.12$ & 0.8553 \\
\hline \multicolumn{7}{|l|}{ Skipping meals } \\
\hline Breakfast & $16.9(0.55)$ & $32.5(0.73)$ & $<0.0001$ & $6.9(1.31)$ & $13.5(3.33)$ & 0.0280 \\
\hline Lunch & $5.5(0.31)$ & $9.3(0.47)$ & $<0.0001$ & $5.5(1.02)$ & $10.0(2.71)$ & 0.0647 \\
\hline Dinner & $3.3(0.24)$ & $5.2(0.32)$ & $<0.0001$ & $4.1(1.11)$ & $2.1(1.04)$ & 0.2319 \\
\hline Eating out (frequency) & & & $<0.0001$ & & & 0.2208 \\
\hline$\geq 0$ nce/day & $35.2(0.70)$ & $42.7(0.80)$ & & $11.6(1.61)$ & $15.0(3.34)$ & \\
\hline 1-6 time(s)/week & $43.4(0.68)$ & $43.0(0.77)$ & & $42.1(2.33)$ & $35.3(4.14)$ & \\
\hline 1-3 time $(\mathrm{s}) /$ month & $14.9(0.45)$ & $10.0(0.43)$ & & $27.9(2.00)$ & $34.5(4.30)$ & \\
\hline$<$ Once/month & $6.5(0.29)$ & $4.2(0.26)$ & & $18.4(1.70)$ & $15.3(2.72)$ & \\
\hline Drinking coffee (frequency) & & & 0.0071 & & & 0.0294 \\
\hline$\geq 0$ nce/day & $71.4(1.40)$ & $77.2(1.30)$ & & $52.6(8.85)$ & $81.9(8.10)$ & \\
\hline 1-6 time(s)/week & $16.1(1.12)$ & $13.1(1.02)$ & & $30.8(8.81)$ & $14.8(7.85)$ & \\
\hline 0-3 time(s)/month & $12.5(1.00)$ & $9.7(0.87)$ & & $16.6(6.32)$ & $3.3(2.50)$ & \\
\hline Perceived health status & & & $<0.0001$ & & & 0.2381 \\
\hline Very good/good & $39.0(0.64)$ & $32.6(0.70)$ & & $24.0(1.90)$ & $18.7(3.64)$ & \\
\hline Fair & $46.6(0.65)$ & $51.2(0.74)$ & & $43.0(2.31)$ & $40.7(4.49)$ & \\
\hline Poor/very poor & $14.4(0.45)$ & $16.3(0.54)$ & & $33.1(2.19)$ & $40.7(4.43)$ & \\
\hline Perceived psychological stress ${ }^{*}$ & $22.0(0.57)$ & $31.8(0.68)$ & $<0.0001$ & $11.6(1.49)$ & $27.3(4.20)$ & $<0.0001$ \\
\hline
\end{tabular}

Values are presented as sample $n$, weighted mean \pm standard error (SE) or weighted percentage (SE): The $p$ values were determined by Student's t-test or Rao-Scott chi-squared test . CKD Stage 3a = eGFR 45-59. CKD Stage 3b = eGFR 30-44. CKD Stage 4-5 = eGFR <30. Drinking coffee was surveyed from 2012-2016 only for the ages of 19-64 years. *The question was: 'How stressed are you on a daily basis?'. The survey participants responded by selecting one of the following responses: 'extremely stressed (severe)', 'quite stressed (moderate)', 'a little bit stressed (mild)', or 'not stressed at all (none)'. In this study, perceived psychological stress was defined as the percentage of participants with severe or moderate stress among all participants. BP: blood pressure. CKD: chronic kidney disease. eGFR: estimated glomerular filtration rate. 
Table 4. Factors associated with quitting smoking in non-CKD and CKD groups

\begin{tabular}{|c|c|c|c|}
\hline Variables & $\begin{array}{c}\text { Non-CKD } \\
\text { AOR }(95 \% \text { CI })\end{array}$ & $\begin{array}{c}\text { CKD } \\
\text { AOR }(95 \% \text { CI })\end{array}$ & Interaction $p *$ \\
\hline Age (years) & & & 0.5481 \\
\hline 19-39 (Ref.) & 1 & 1 & \\
\hline $40-64$ & $2.07(1.83-2.34)$ & $1.20(0.21-6.73)$ & \\
\hline$\geq 65$ & $4.82(3.99-5.82)$ & $2.27(0.41-12.57)$ & \\
\hline Marital status (married) & $2.07(1.82-2.35)$ & $0.86(0.43-1.71)$ & 0.0139 \\
\hline Occupation & & & 0.1587 \\
\hline Manager/professionals (Ref.) & 1 & 1 & \\
\hline Office worker & $1.01(0.85-1.19)$ & $6.10(1.48-25.11)$ & \\
\hline Service workers/sellers & $0.86(0.72-1.02)$ & $1.67(0.45-6.22)$ & \\
\hline Agriculture/fishery/labor & $0.94(0.81-1.09)$ & $2.46(0.85-7.13)$ & \\
\hline None & $0.94(0.81-1.09)$ & $2.52(0.97-6.54)$ & \\
\hline Household income (\% in quartiles) & & & 0.1743 \\
\hline 1st (lowest) (Ref.) & 1 & 1 & \\
\hline 2nd & $1.14(1.00-1.30)$ & $1.76(0.92-3.38)$ & \\
\hline 3rd & $1.16(1.02-1.33)$ & $2.04(1.05-3.96)$ & \\
\hline 4th (highest) & $1.33(1.16-1.53)$ & $2.67(1.32-5.42)$ & \\
\hline Lifetime smoking amount (each 10 pack-year increase) & $0.86(0.83-0.88)$ & $1.06(0.97-1.16)$ & $<0.0001$ \\
\hline \multicolumn{4}{|l|}{ Secondhand smoke exposure } \\
\hline Workplace (yes) & $0.84(0.76-0.94)$ & $0.79(0.38-1.66)$ & 0.8592 \\
\hline Home (yes) & $0.48(0.37-0.63)$ & $1.91(0.53-6.91)$ & 0.0396 \\
\hline \multicolumn{4}{|l|}{ Smoking cessation methods } \\
\hline Willpower & $1.53(1.28-1.83)$ & $1.58(0.63-3.93)$ & 0.9509 \\
\hline Nicotine replacement therapy & $0.46(0.38-0.55)$ & $0.57(0.22-1.50)$ & 0.6617 \\
\hline Education/counselling & $0.55(0.46-0.66)$ & $0.41(0.17-0.99)$ & 0.5304 \\
\hline Smokers' quitline & $0.74(0.45-1.21)$ & $0.56(0.10-3.16)$ & 0.7619 \\
\hline \multicolumn{4}{|l|}{ Health status } \\
\hline eGFR, per 10-unit increase & $0.91(0.88-0.95)$ & $0.91(0.70-1.17)$ & 0.9472 \\
\hline Systolic blood pressure (each 10-mmHg increase) & $1.07(1.02-1.11)$ & $0.89(0.79-1.01)$ & 0.0067 \\
\hline Body mass index $\left(\mathrm{kg} / \mathrm{m}^{2}\right)$ & $1.04(1.02-1.06)$ & $1.07(0.98-1.17)$ & 0.5297 \\
\hline Fasting blood sugar (each 10 mg/dL increase) & $1.03(1.00-1.05)$ & $0.99(0.92-1.07)$ & 0.3579 \\
\hline Hypertension & $1.19(1.05-1.34)$ & $1.09(0.64-1.86)$ & 0.7579 \\
\hline Diabetes mellitus & $0.86(0.71-1.04)$ & $0.79(0.46-1.35)$ & 0.7560 \\
\hline Cardiovascular disease & $1.22(0.97-1.53)$ & $1.96(1.04-3.69)$ & 0.1666 \\
\hline Cancer & $1.98(1.47-2.65)$ & $1.95(0.72-5.23)$ & 0.9765 \\
\hline Trauma history & $0.84(0.71-1.00)$ & $1.57(0.60-4.15)$ & 0.2150 \\
\hline Heavy drinker & $0.68(0.61-0.76)$ & $1.17(0.49-2.83)$ & 0.2279 \\
\hline Regular exercise & $1.29(1.17-1.42)$ & $2.84(1.52-5.31)$ & 0.0153 \\
\hline \multicolumn{4}{|l|}{ Skipping meals } \\
\hline Breakfast & $0.64(0.57-0.73)$ & $0.62(0.27-1.46)$ & 0.9427 \\
\hline Lunch & $0.68(0.56-0.82)$ & $0.41(0.14-1.22)$ & 0.3758 \\
\hline Dinner & $0.78(0.63-0.98)$ & $3.23(0.68-15.28)$ & 0.0754 \\
\hline Eating out (frequency) & & & 0.1211 \\
\hline$\geq 0$ nce/day (Ref.) & 1 & 1 & \\
\hline 1-6 time(s)/week & $1.02(0.92-1.14)$ & $1.30(0.56-2.98)$ & \\
\hline 1-3 time(s)/month & $1.08(0.92-1.27)$ & $0.63(0.27-1.45)$ & \\
\hline$<$ Once/month & $0.93(0.75-1.15)$ & $0.76(0.33-1.78)$ & \\
\hline Perceived health status & & & 0.9139 \\
\hline Very good/good & $1.44(1.24-1.66)$ & $1.24(0.61-2.55)$ & \\
\hline Fair & $1.12(0.98-1.28)$ & $1.02(0.59-1.79)$ & \\
\hline Poor/very poor (Ref.) & 1 & 1 & \\
\hline Perceived psychological stress & $0.79(0.71-0.88)$ & $0.46(0.25-0.87)$ & 0.1061 \\
\hline
\end{tabular}

${ }^{*}$ p-values were obtained by the Wald test with logistic regression. Cl: confidence interval. CKD: chronic kidney disease. eGFR: estimated glomerular filtration rate. AOR: adjusted odds ratio. 


\section{Sociodemographic characteristics and smoking history}

Table 2 shows the participants' sociodemographic characteristics and smoking history. In the nonCKD group, quitters were more likely to be older, married, and have higher incomes than non-quitters. Secondhand smoke exposure either at the workplace or at home was more common in non-quitters. As for the smoking cessation method, quitters used willpower more often, whereas non-quitters chose nicotine replacement therapy (NRT), education/ counselling, and quitlines more frequently.

In the CKD group, quitters were more likely to be older and have higher incomes. The lifetime smoking amount in quitters was 28.2 pack-years, significantly higher than the 25.0 pack-years in non-quitters $(p=0.0439)$. Secondhand smoke exposure at the workplace was more common in non-quitters. Quitters used willpower more often regarding the smoking cessation method, whereas non-quitters chose NRT and education/counselling more frequently.

\section{Clinical characteristics and health behaviors and perceptions}

Table 3 displays the participants' clinical characteristics, health behaviors, and perceptions, for both groups. In the non-CKD group, blood pressure, body mass index, waist circumference, and fasting blood sugar were higher in quitters. Comorbidities such as hypertension, diabetes mellitus, CVD, and cancer prevalence were more often observed in quitters. Quitters exercised regularly and more frequently. In contrast, trauma history, heavy drinking, skipping meals (breakfast, lunch, or dinner), eating out, and drinking coffee more than once per day, poor perceived health status, and high perceived psychological stress were more often observed in non-quitters.

CVD, cancer prevalence, and regular exercise were more frequently observed in the CKD group in quitters. Skipping breakfast, drinking coffee more than once per day, and high perceived psychological stress were more common among non-quitters.

\section{Factors associated with smoking cessation}

Table 4 shows the factors associated with quitting smoking and the interaction effects between the nonCKD and CKD groups. Additionally, Figure 2 shows the significant factors and p-values for the interactions between the two groups.

Regular exercise was the only factor that was

Figure 2. Significant factors associated with quitting smoking in non-CKD and CKD groups with $p$ for the interactions between two groups
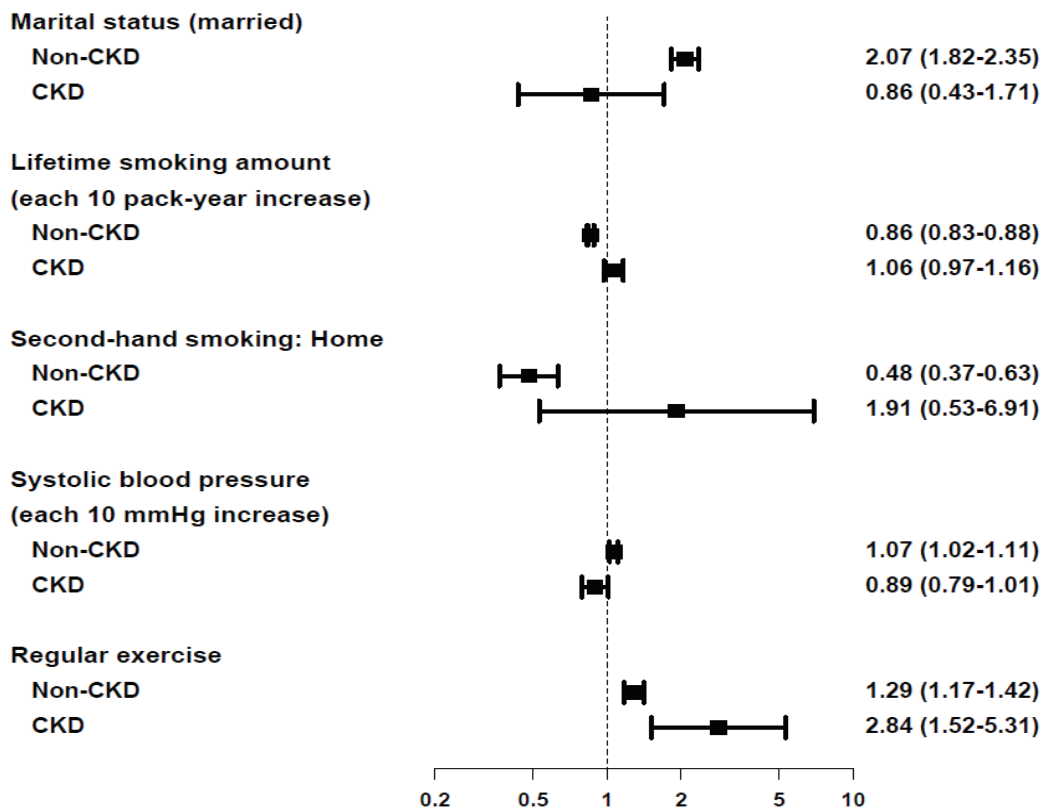

CKD: chronic kidney disease. OR: odds ratio. CI: confidence interval.

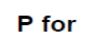


significantly associated with quitting in both nonCKD and CKD groups, indicating that people who exercise regularly have a 1.29 -fold and 2.84-fold increase in quitting success rate in the non-CKD and the CKD groups, respectively (adjusted odds ratio, $\mathrm{AOR}=1.29 ; 95 \% \mathrm{CI}: 1.17-1.42$ for the non-CKD group; $\mathrm{AOR}=2.84$; $95 \%$ CI: $1.52-5.31$ for the CKD group, interaction $\mathrm{p}=0.0153)$. Unlike in the CKD group, a few more variables were also associated with quitting in the non-CKD group. Marriage and higher systolic blood pressure (SBP) were associated with quitting only in the non-CKD group (marriage: $\mathrm{AOR}=2.07$; 95\% CI: $1.84-2.39$, interaction $\mathrm{p}=0.0139$; SBP: $A O R=1.07 ; 95 \%$ CI: $1.02-1.11$, interaction $\mathrm{p}=0.0067$ ), while lifetime smoking amount and secondhand smoke exposure at home were negatively associated with quitting smoking in the non-CKD group (lifetime smoking amount: $\mathrm{AOR}=0.86$; $95 \% \mathrm{CI}$ : $0.83-0.88$, interaction $\mathrm{p}<0.0001$; secondhand smoke exposure at home: $\mathrm{AOR}=0.48$; 95\% CI: $0.37-0.63$, interaction $\mathrm{p}=0.0396$ ).

\section{DISCUSSION}

This study aimed to examine and compare factors associated with quitting smoking in Korean adult male smokers, with and without CKD, using KNHANES data. The findings can be summarized as follows: 1) smoking cessation rates in the CKD group remained relatively stable during the study period, whereas those in the non-CKD group tended to increase; 2 ) fewer factors were significant in quitting smoking in the CKD group than in the non-CKD group; and $3)$ the interaction effects between the two groups revealed that regular exercise was the only factor associated with quitting in the CKD group.

As stated above, smoking cessation rates in participants without CKD tended to increase over the study period; this has been previously observed in Korean male smokers (Table 1$)^{30}$. During the study period, cessation rates in the CKD group did not seem to increase but were higher than those in the non-CKD group throughout the study. It has been reported that smokers with chronic medical conditions attempt to quit smoking and use evidencebased tobacco-cessation treatment more frequently than smokers without comorbidities. Smoking in individuals with chronic medical conditions can negatively affect symptoms, disease progression, and mortality. Therefore, chronic comorbidities can often motivate quitting smoking, but it is controversial whether this motivation leads to quitting g $^{30,32,33}$. However, our study findings suggest that CKD may have motivated patients to quit. Another possibility is that older age is associated with a higher success rate of quitting smoking among Korean adult males, as has been reported from previous national surveys. Given that the CKD group is older than the non-CKD group, this age difference might explain the higher smoking cessation rate in the CKD group ${ }^{30,33,34}$.

In the CKD group, significant differences were observed in fewer clinical characteristics between quitter and non-quitters than in the non-CKD group (Tables 2 and 3). For instance, among quitters in the non-CKD group, most comorbid conditions were common, but only cardiovascular comorbidity and cancer were frequently observed among those in the CKD group. This may be simply because of the higher prevalence of CVD and cancer in patients with CKD than in the general population, as they are the leading cause of death in the CKD population ${ }^{1,3,4,35}$. However, the explanation of this finding, i.e. fewer factors associated with quitting in the CKD group, remains unclear. It has been reported that patients with CKD tend to share many commonalities in socioeconomic status, demographic characteristics, health perception and behavior, and comorbidities, which might explain the small number of differences between two groups (quitter vs non-quitter) in patients with $\mathrm{CKD}^{3}$.

When the interaction effects between groups were evaluated, regular exercise was the only factor associated with cessation in the CKD group (Table 4 and Figure 2). Exercise was associated with quitting in the non-CKD group as well; however, based on the AOR, exercise had a more significant effect on smoking cessation in the CKD than in the non-CKD group. Despite extensive research on the importance of exercise in smoking cessation, there does not seem to be a consensus on its effect ${ }^{36-38}$. Regular exercise is expected to contribute to smoking cessation by attenuating cigarette withdrawal symptoms and cravings or controlling weight ${ }^{36,37}$. A systematic review suggests that aerobic exercise may be effective in quitting smoking in the short-term, but not in the medium-term to long-term follow-up ${ }^{37}$. However, another review reports no effect of exercise on smoking cessation, regardless of the type of exercise ${ }^{38}$. 
In the non-CKD group, the interaction effects revealed the associations of several other variables with smoking cessation. Marriage and SBP were associated with quitting, while secondhand smoke exposure and greater lifetime smoking were negatively associated with quitting. Previous national surveys have reported older age, being married, higher education level, using willpower to quit, alcohol abstinence, smokefree environment, or better stress management, as successful smoking cessation factors in Korean adult males. Therefore, our study findings are consistent with existing national surveys investigating smoking cessation success factors in Korean male adults ${ }^{30,33,34}$.

This study has examined a wide range of potential predictors of quitting smoking, such as 'skipping meals' or 'frequency of eating out'. We selected these variables as potential predictors for the following reasons: 1) the study aim was to find the diseasespecific factors associated with quitting status. CKD is a disease in which dietary precautions are critical to modify disease progression. It is recommended for patients with CKD to eat regularly and dine out less frequently ${ }^{39}$; 2) 'meal skipping' or 'frequent eating out' were previously reported as predictors of smoking cessation failure ${ }^{30}$. Viewed in combination, we speculated that these variables could represent overall treatment adherence, including smoking cessation, in patients with CKD. Therefore, we included these variables in the analysis.

\section{Limitations}

There are a few limitations to this study. The reliability of smoking history may be lower because the survey was conducted only with self-report. Although we analyzed a comprehensive set of potential predictors of smoking cessation, we did not include some important, evidence-based predictors in the analysis. For example, the analysis did not include levels of tobacco dependence and motivation to quit smoking, history of using e-cigarettes or history of mental disorders such as anxiety or depression. The reason for this was that the KNHANES does not provide such information. In particular, people with mental illnesses tend to be more heavily addicted to nicotine when they smoke, have greater difficulty quitting, but are less likely to get help with quitting than the general population of smokers. Regrettably, mental illness could not be analyzed in this study ${ }^{40}$.
Notably, a diagnosis of CKD requires maintaining chronicity of reduced eGFR for at least three months However, as the KNHANES is a cross-sectional survey, we could not confirm the chronicity of kidney dysfunction in the study participants. Therefore, some participants with acute kidney injury might have been included in the CKD group ${ }^{26,27}$.

Lastly, our study adopted a cross-sectional design with the associated limitations in interpreting the results. Thus, further studies should address the causal relationship between exercise and smoking cessation in the population with GKD to examine the replicability of our study findings, particularly since no previous studies have examined disease-specific smoking cessation factors.

\section{CONCLUSIONS}

Exercise was the only factor associated with quitting smoking in the CKD group. Further analysis using a longitudinal design to determine the role of exercise in smoking cessation in the CKD population will be needed. This study showed that participants with CKD differed in factors associated with quitting smoking compared to participants without CKD. This may imply a need for disease-specific approach to improving the effectiveness of smoking cessation.

\section{REFERENCES}

1. National Kidney Foundation. K/DOQI clinical practice guidelines for chronic kidney disease: evaluation, classification, and stratification. Am J Kidney Dis. 2002;39(2 Suppl 1):S1-266. Accessed November 30, 2021. https://www.kidney.org/sites/default/files/docs/ ckd_evaluation_classification_stratification.pdf

2. GBD Chronic Kidney Disease Collaboration. Global, regional, and national burden of chronic kidney disease, 1990-2017: a systematic analysis for the Global Burden of Disease Study 2017. Lancet. 2020;395(10225):709-733. doi:10.1016/S0140-6736(20)30045-3

3. Snyder JJ, Collins AJ. Association of preventive health care with atherosclerotic heart disease and mortality in CKD. J Am Soc Nephrol. 2009;20(7):1614-1622. doi:10.1681/ASN.2008090954

4. Sarnak MJ, Levey AS, Schoolwerth AC, et al. Kidney disease as a risk factor for development of cardiovascular disease: a statement from the American Heart Association Councils on Kidney in Cardiovascular Disease, High Blood Pressure Research, Clinical Cardiology, and Epidemiology and Prevention. Hypertension. 2003;108(17):2154-2169. doi:10.1161/01.CIR.0000095676.90936.80

5. Roy A, Rawal I, Jabbour S, Prabhakaran D. Tobacco 
and Cardiovascular Disease: A Summary of Evidence. In: Cardiovascular, Respiratory, and Related Disorders. 3rd ed. The International Bank for Reconstruction and Development, The World Bank; 2017. doi:10.1596/978-1-4648-0518-9_ch4

6. Sarnak MJ, Greene T, Wang X, et al. The effect of a lower target blood pressure on the progression of kidney disease: long-term follow-up of the modification of diet in renal disease study. Ann Intern Med. 2005;142(5):342351. doi:10.7326/0003-4819-142-5-200503010-00009

7. Eriksen BO, Ingebretsen OC. The progression of chronic kidney disease: a 10-year population-based study of the effects of gender and age. Kidney Int. 2006;69(2):375382. doi:10.1038/sj.ki.5000058

8. Hallan SI, Coresh J, Astor BC, et al. International comparison of the relationship of chronic kidney disease prevalence and ESRD risk. J Am Soc Nephrol. 2006;17(8):2275-2284. doi:10.1681/ASN.2005121273

9. Orth SR, Hallan SI. Smoking: a risk factor for progression of chronic kidney disease and for cardiovascular morbidity and mortality in renal patients--absence of evidence or evidence of absence? Clin J Am Soc Nephrol. 2008;3(1):226-236. doi:10.2215/CJN.03740907

10. Bleyer AJ, Shemanski LR, Burke GL, Hansen KJ, Appel RG. Tobacco, hypertension, and vascular disease: risk factors for renal functional decline in an older population. Kidney Int. 2000;57(5):2072-2079. doi:10.1046/j.1523-1755.2000.00056.x

11. de Boer IH, Sibley SD, Kestenbam B, et al. Central obesity, incident microalbuminuria, and change in creatinine clearance in the epidemiology of diabetes interventions and complications study. J Am Soc Nephrol. 2007;18(1):235-243. doi:10.1681/ASN.2006040394

12. Wonji J, Sangmi L, Su JY, et al., Association of smoking with incident CKD risk in the general population: A communitybased cohort study. PLoS One. 2020;15(8):e0238111. doi:10.1371/journal.pone.0238111

13. Staplin N, Haynes R, Herrington WG, et al. Smoking and Adverse Outcomes in Patients With CKD: The Study of Heart and Renal Protection (SHARP). Am J Kidney Dis. 2016;68(3):371-380. doi:10.1053/j.ajkd.2016.02.052

14. Hogan SL, Vupputuri S, Guo X, et al. Association of cigarette smoking with albuminuria in the United States: the third National Health and Nutrition Examination Survey. Ren Fail. 2007;29(2):133-142. doi:10.1080/08860220601098888

15. Sangmi L, Shinchan K, Su JY, et al. Smoking, Smoking Cessation, and Progression of Chronic Kidney Disease: Results From KNOW-CKD Study. Nicotine Tob Res. 2021;23(1):92-98. doi:10.1093/ntr/ntaa071

16. Ohkuma T, Nakamura U, Iwase M, et al. Effects of smoking and its cessation on creatinine- and cystatin C-based estimated glomerular filtration rates and albuminuria in male patients with type 2 diabetes mellitus: the Fukuoka Diabetes Registry. Hypertens Res. 2016;39(10):744-751. doi:10.1038/hr.2016.51

17. Formanek P, Salisbury-Afshar E, Afshar M. Helping Patients With ESRD and Earlier Stages of CKD to Quit Smoking. Am J Kidney Dis. 2018;72(2):255-266. doi:10.1053/j.ajkd.2018.01.057

18. Filozof C, Fernandez Pinilla MC, Fernandez-Cruz A. Smoking cessation and weight gain. Obes Rev. 2004;5(2):95-103. doi:10.1111/j.1467-789X.2004.00131.x

19. Ockene JK, Mermelstein RJ, Bonollo DS, et al. Relapse and maintenance issues for smoking cessation. Health Psychol. 2000;19(1S):17-31. doi:10.1037/0278-6133.19.Suppl1.17

20. Abdul-Kader J, Airagnes G, D'Almeida S, Limosin F, le Faou AL. [Interventions for smoking cessation in 2018]. Rev Pneumol Clin. 2018;74(3):160-169. doi:10.1016/j.pneumo.2018.03.004

21. Abrams D, Niaura R, Brown RA, Emmons KM, Goldstein MG, Monti PR. The Tobacco Dependence Treatment Handbook: A Guide to Best Practices. Guilford; 2003.

22. Korea Disease Control and Prevention Agency. Korea National Health and Nutrition Examination Survey. Accessed April 9, 2021. https://knhanes.kdca.go.kr/ knhanes/eng/index.do

23. Gunter R, Sxeto E, Jeong SH, Suh S, Waters AJ. Cigarette Smoking in South Korea: A Narrative Review. Korean J Fam Med. 2020;41(1):3-13. doi:10.4082/kjfm.18.0015

24. Kang HG, Kwon KH, Lee IW, Jung B, Park EC, Jang SI. Biochemically-verified smoking rate trends and factors associated with inaccurate self-reporting of smoking habits in Korean women. Asian Pac J Cancer Prev. 2013;14(11):68076812. doi:10.7314/APJCP.2013.14.11.6807

25. Jung-Choi KH, Khang YH, Cho HJ. Hidden female smokers in Asia: a comparison of self-reported with cotinine-verified smoking prevalence rates in representative national data from an Asian population. Tob Control. 2012;21(6):536542. doi:10.1136/tobaccocontrol-2011-050012

26. Levey AS, Coresh J, Greene T, et al. Expressing the Modification of Diet in Renal Disease Study equation for estimating glomerular filtration rate with standardized serum creatinine values. Clin Chem. 2007;53(4):766-772. doi:10.1373/clinchem.2006.077180

27. Levin A, Stevens PE. Summary of KDIGO 2012 CKD Guideline: behind the scenes, need for guidance, and a framework for moving forward. Kidney Int. 2014;85(1):49-61. doi:10.1038/ki.2013.444

28. Centers for Disease Control and Prevention. National Health Interview Survey: Glossary. Updated August 29, 2017. Accessed March 12, 2021. https://www.cdc.gov/ nchs/nhis/tobacco/tobacco_glossary.htm

29. Pomerleau CS, Pomerleau OF, Snedecor SM, Mehringer AM. Defining a never-smoker: results from the nonsmokers survey. Addict Behav. 2004;29(6):11491154. doi:10.1016/j.addbeh.2004.03.008

30. Kim Y, Lee JS, Cho WK. Factors Associated with Successful Smoking Cessation According to Age Group: Findings of an 11-Year Korea National Survey. 
Int J Environ Res Public Health. 2021;18(4):1576. doi:10.3390/ijerph18041576

31. Kweon S, Kim Y, Jang MJ, et al. Data resource profile: the Korea National Health and Nutrition Examination Survey (KNHANES). Int J Epidemiol. 2014;43(1):69-77. doi:10.1093/ije/dyt228

32. Rojewski AM, Baldassarri S, Cooperman NA, et al. Exploring Issues of Comorbid Conditions in People Who Smoke. Nicotine Tob Res. 2016;18(8):1684-1696. doi:10.1093/ntr/ntw016

33. Kim Y, Cho WK. Factors Associated with Successful Smoking Cessation in Korean Adult Males: Findings from a National Survey. Iran J Public Health. 2014;43(11):14861496. Accessed November 30, 2021. https://www.ncbi. nlm.nih.gov/labs/pmc/articles/PMC4449497/

34. Kim YJ. Predictors for successful smoking cessation in Korean adults. Asian Nurs Res (Korean Soc Nurs Sci). 2014;8(1):1-7. doi:10.1016/j.anr.2013.09.004

35. Malyszko J, Tesarova P, Capasso G, Capasso A. The link between kidney disease and cancer: complications and treatment. Lancet. 2020;396(10246):277-287. doi:10.1016/S0140-6736(20)30540-7

36. Ussher MH, Faulkner GEJ, Angus K, Hartmann-Boyce J, Taylor AH. Exercise interventions for smoking cessation. Cochrane Database Syst Rev. 2019;2019(10). doi:10.1002/14651858.CD002295.pub6

37. Santos CP, Proenca M, Gouveia TdS, et al. Effectiveness of Aerobic Exercise on Smoking Cessation in Adults: A Systematic Review and Meta-Analysis. J Phys Act Health. 2021;18(2):230-242. doi:10.1123/jpah.2019-0339

38. Klinsophon T, Thaveeratitham P, Sitthipornvorakul E, Janwantanakul P. Effect of exercise type on smoking cessation: a meta-analysis of randomized controlled trials. BMC Res Notes. 2017;10(1):442. doi:10.1186/s13104-017-2762-y

39. Kramer H. Diet and Chronic Kidney Disease. Adv Nutr. 2019;10(Suppl_4):S367-S379.doi:10.1093/advances/nmz011

40. Gilbody S, Peckham E, Bailey D. Smoking cessation for people with severe mental illness (SCIMITAR+): a pragmatic randomised controlled trial. Lancet Psychiatry. 2019;6(5):379-390. doi:10.1016/S2215-0366(19)30047-1
CONFLICTS OF INTEREST

The authors have completed and submitted the ICMJE Form for Disclosure of Potential Conflicts of Interest and none was reported.

\section{FUNDING}

There was no source of funding for this research.

\section{ETHICAL APPROVAL AND INFORMED CONSENT}

The study was conducted according to the guidelines of the Declaration of Helsinki. Ethical approval and informed consent were not required, as the current study used only existing de-identified data from the Korea National Health and Nutrition Examination Survey (KNHANES). KHANES Survey was reviewed and approved by the Korea Centers for Disease Control and Prevention (IRB approval numbers were 2008-04EXP01-C, 2009-01CON-03-2C, 2010-02CON-21-C, 2011-02CON-06-C, 2012-01EXP-01-2C, 2013-12EXP-03-5C, 2018-01-03-P-A, and 201801-03-C-A).

\section{DATA AVAILABILITY}

The data supporting this research are available from the following sources: http://knhanes.cdc.go.kr/

\section{AUTHORS' CONTRIBUTIONS}

Conceptualization, YK and WKC; Methodology, YK and WKC; Formal analysis, YK; Investigation, YK and WKC; Data curation, YK and WKC; Writing and original draft preparation $Y K$; Writing, review and editing, WKC; Supervision, WKC.

\section{PROVENANCE AND PEER REVIEW}

Not commissioned; externally peer reviewed. 